\title{
Interação espécie-ambiente na colonização de uma voçoroca em Diamantina, Minas Gerais
}

\author{
Paula Alves Oliveira ${ }^{1}$ Keila Cristina Vieira ${ }^{1}$ Israel Marinho Pereira ${ }^{1}$ Marcio Leles Romarco de Oliveira $^{1}$ Leonardo \\ Palhares Silveira $^{1}$ Evandro Luiz Mendonça Machado ${ }^{1}$ Wellington Willian Rocha ${ }^{1}$
}

${ }^{1}$ Universidade Federal dos Vales do Jequitinhonha e Mucuri, Rodovia MGT 367, Km 583, 5000 - Alto da Jacuba, Diamantina - MG, 39100 -000

*Author for correspondence: oliveiraalvespaula@gmail.com

Received: December 2018 / Accepted: September 2019/ Published: September 2019

\section{Resumo}

A voçoroca é considerada o estágio mais avançado da erosão hídrica e uma das maiores causas de perda da camada superficial do solo. $\mathrm{O}$ objetivo do presente trabalho foi conhecer e avaliar a composição florística, a estrutura e a diversidade das espécies presentes na colonização de uma voçoroca inserida no bioma cerrado e verificar a influência do ambiente na distribuição e ocorrência desses indivíduos. A área foi estratificada em três estratos (ambientes), definidos em função do estágio de sucessão em que se encontram e das características do substrato como: ambiente I ou baixada; ambiente II ou parte intermediária; ambiente III ou superior. No ambiente III foi registrado o maior número de espécies, isto sugere que o mesmo encontre em fase mais avançada de sucessão. $\mathrm{O}$ dendrograma gerado pela análise de agrupamentos evidencia uma separação das parcelas em dois grandes grupos, o primeiro, formado pelas parcelas do ambiente I e parte das parcelas do ambiente II. Já o segundo, foi formado pelas parcelas do ambiente III e parte do ambiente II.

Palavras-chave: degradação, espécies indicadoras, regeneração, sucessão

\section{Abstract}

The purpose of this study was to observe and evaluate the floristic composition, structure and diversity of species in the colonization of a gully in the cerrado biome and the influence of the environment on the distribution and occurrence of these species. The area was divided into three environments, defined according to the position they are in and the substrate characteristics: environment I or lowland; environment II or intermediate part; environment III or upland. The highest number of species recorded in environment III suggested an advanced stage of succession. The dendrogram generated by cluster analy sis shows a separation of the plots in two groups, the first, formed by plots of environment I and part of those of environment II. The second consisted of plots of environment III and part of those of environment II. Keywords: degradation, indicator species, regeneration, succession

\section{Introdução}

A erosão hídrica é uma das maiores causas de perda da camada superficial do solo (Abdo et al. 2008), a qual ocasiona redução da cobertura vegetal, acidificação do solo, diminuição do teor de carbono orgânico e da biodiversidade (Carneiro et al. 2009). Dentre as mais diferentes categorias de erosão hídrica, a voçoroca é considerada o estágio mais avançado deste tipo de erosão (Ferreira et al. 2011), causando a degradação do solo e comprometendo grandes áreas (Gomide et al. 2011). Vários são os fatores que controlam o processo de voçorocamento e estão intimamente ligados, tais como: os solos, o uso e manejo do solo, topografia, litologia e a vegetação que atua no controle da erosão causada por ações antrópicas ou naturais (Gutiérrez et al. 2009; Ferreira et al. 2011).

A falta de conhecimento científico sobre o ecossistema do cerrado contribuiu para uma ocupação desordenada, e consequentemente em um intenso processo de desmatamento deste bioma (Lanza et al., 2014). A maior parte da produção nacional de grãos está localizada no cerrado (Sano et al., 2010), o que culminou, nos últimos anos, numa taxa de desmatamento superior à da região amazônica (Sano et al., 2019). Grande parte da área natural deste bioma enfrenta degradação devido à extração seletiva insustentável (Ferreira et al., 2012), além disso, possui solos arenosos, os quais estão mais sujeitos à erosão e posterior formação de voçorocas (Goulart e Azevedo, 2015).

O Parque Estadual do Biribiri (PEB), onde está inserida área de trabalho, se localiza na Serra do Espinhaço Meridional e possui inúmeras áreas que já foram garimpadas e que atualmente se encontram abandonadas (Amaral et al. 2013), das quais muitas possuem voçorocamento. A principal bacia detentora dos cursos d'água do PEB é a do Rio Jequitinhonha, que ao norte marca o limite do parque pela sua confluência com o Rio Pinheiros (IEF, 2005). O PEB possui alto índice de táxons endêmicos (Echternacht et al. 2011), e suas fitofisionomias predominantes são: formações savânicas (cerrados sentido restrito e rupestre), campestres (campos limpos, úmidos e rupestres) e florestais (florestas estacionais semideciduais), constituindo um ecótono entre o Cerrado e a Mata Atlântica. Contudo, é marcante a presença de áreas em diferentes estágios de regeneração pós-distúrbio (Pereira et al. 2015).

A vegetação é um fator determinante na dinâmica dos processos erosivos (Bonini et al. 2013), sendo, portanto, necessário o conhecimento sobre as espécies que compõem a flora local, bem como a sua diversidade no ambiente degradado. Segundo Loschi et al. (2011), os estudos que buscam conhecer os atributos do solo, sua composição florística e as características ecológicas das espécies ocorrentes em ambientes alterados por processos de voçorocamento têm muito a contribuir com programas que visam a conservação, a preservação e a recuperação desses ecossistemas.

Portanto, para um melhor entendimento do funcionamento ecológico dessas espécies, assim como, para a proposição de medidas de recuperação e conservação destes ambientes. Assim, a fitossociologia se destaca como ferramenta indispensável, já que permiti inferir sobre as espécies presentes, sua proporção, distribuição e se associação (Messias et al. 2012).

Diante disso, o presente trabalho objetivou avaliar a composição florística, estrutura e diversidade das espécies em regeneração numa voçoroca localizada no município de Diamantina, Minas Gerais, verificando ainda a influência do solo na distribuição e ocorrência destas espécies. 


\section{Material e Métodos}

$\mathrm{O}$ presente estudo encontra-se no $\mathrm{PEB}$, município de Diamantina, MG. O Parque possui área de 16.998,66 hectares e está situado entre as coordenadas geográficas $18^{\circ} 14^{\prime} 53^{\prime}$ S e $43^{\circ} 39^{\prime} 57^{\prime \prime} \mathrm{W}$ e $18^{\circ} 02^{\prime} 15^{\prime \prime} \mathrm{S}$ e $43^{\circ} 29^{\prime} 36^{\prime \prime} \mathrm{W}$, região do Alto Vale do Rio Jequitinhonha, no Complexo da Serra do Espinhaço Meridional. $\mathrm{O}$ solo predominante da região é o neossolo litólico psamítico típico (RLq), que é raso, pedregoso, com permeabilidade moderada, baixa fertilidade e baixa capacidade de saturação e de retenção de água (IEF 2005).

O regime climático da região é tipicamente tropical, $\mathrm{Cwb}$ na classificação de Koppen, caracterizado por verões brandos e úmidos (outubro a abril) e invernos mais frescos e secos (junho a agosto) (Sá Júnior et al. 2012). A precipitação média anual varia de 1.250 a $1.550 \mathrm{~mm}$ e a temperatura média anual situa-se na faixa de 18 a $19{ }^{\circ} \mathrm{C}$. A umidade relativa do ar é quase sempre elevada, revelando médias anuais de 75,6\%.

Para o estudo fitossociológico das espécies colonizadoras, a voçoroca foi estratificada em três estratos (ambientes), os quais foram definidos em função do estágio de sucessão em que se encontram e das características do substrato, assim caracterizados: ambiente I ou baixada: ambiente com maior teor de umidade e solo mais arenoso ( $8,40 \%$ de argila) e menores teores de matéria orgânica $(0,71$ dak $\mathrm{kg}^{-1}$ ) e com maior diversidade aparente de espécies; ambiente II ou parte intermediária: área com declividade, umidade e teores de matéria orgânica $\left(1,52 \mathrm{dak} \mathrm{kg}^{-1}\right)$ e argila $(16,7 \%)$ intermediários, caracterizados como transição entre os ambientes I e III e; ambiente III ou superior: ambiente que possui solos mais profundos, com maiores teores de argila $(26,5 \%)$ e matéria orgânica $\left(3,10 \mathrm{dak} \mathrm{kg}^{-1}\right)$ e mais declivoso.

Para a amostragem da vegetação foram utilizadas parcelas distribuídas de forma sistemática a cada 5 metros. Foram alocadas 36 parcelas de $3 \times 5 \mathrm{~m}\left(15 \mathrm{~m}^{2}\right)$ nos três ambientes. Mensurou-se altura e diâmetro de todos os indivíduos com altura $\geq 0,10 \mathrm{~m}$ e diâmetro à altura do solo $(\mathrm{DAS}) \leq 5,0 \mathrm{~cm}$ que posteriormente, foram classificados em três classes de tamanho de regeneração natural, segundo metodologia recomendada por Finol (1971) e adaptada para este trabalho. As classes de tamanho adotadas foram: Classe I: indivíduos com altura entre $0,1 \mathrm{~m}$ e $0,3 \mathrm{~m}$; Classe II: indivíduos com altura entre $0,31 \mathrm{~m}$ e $1,50 \mathrm{~m}$ e; Classe III: indivíduos com altura superior a $1,51 \mathrm{~m}$.

Foram coletados representantes de todas as espécies amostradas no período de agosto de 2007 a setembro de 2008 . $\mathrm{O}$ material fértil coletado foi depositado e identificado no Herbário Dendrológico Jeanine Felfili (HDJF) da Universidade Federal dos Vales do Jequitinhonha e Mucuri (UFVJM). As espécies foram classificadas em famílias de acordo com o sistema Angiosperm Phy logeny Group (APG IV, 2016) e a grafia dos nomes foi confirmada no banco Flora do Brasil < http://floradobrasil.jbrj.gov.br>.

A diversidade e riqueza das espécies arbustivo-arbóreas na amostra total e nos diferentes ambientes foram avaliadas pelos seguintes parâmetros: riqueza de espécies, densidade de espécies por parcela e índices de diversidade de Shannon $\left(\mathrm{H}^{\prime}\right)$ e Indice de similaridade de Jaccard (Brower e Zar, 1984). Os índices de diversidade de Shannon entre os três ambientes foram comparados pelo teste t de Hutcheson (Zar, 1996). Adicionalmente, no sentido de reconhecer o compartilhamento e comparação das plantas, foi produzido um diagrama de Venn com as espécies encontradas (Oliveros, 2007).

Foi utilizada a Análise de Espécies Indicadoras (Dufrêne e Legendre, 1997) processada pelo programa PC-ORD for Windows versão 4.14 (Mccune e Mefford, 1999) como ferramenta na determinação das preferências das espécies pelos três ambientes pré-determinados.

\section{Resultados e Discussão}

No presente estudo foram registrados 1382 indivíduos, representados por 40 famílias, 80 gêneros e 131 espécies (Tabela 1). As famílias que apresentaram o maior número de espécies foram: Myrtaceae (27); Melastomataceae (18); Asteraceae (12); Rubiaceae (7) e Malpighiaceae (7). Juntas essas famílias, contribuíram com 53,78\% do total de espécies amostradas.

Tabela 1. Lista das espécies arbustivo-arbórea registradas nos três ambientes de uma voçoroca no PEP em Diamantina, MG, dispostas em ordem alfabética de famílias e acompanhadas dos parâmetros fitossociológicos. $\mathrm{NI}=$ número de indivíduos; IVI = índice de valor de importância (\%)

\begin{tabular}{|c|c|c|c|c|c|c|}
\hline \multirow{2}{*}{ Família/Espécies } & \multicolumn{2}{|c|}{ Ambiente I } & \multicolumn{2}{|c|}{ Ambiente II } & \multicolumn{2}{|c|}{$\underset{\text { III }}{\text { Ambiente }}$} \\
\hline & NI & IVI & NI & IVI & NI & IVI \\
\hline \multicolumn{7}{|l|}{ ANNONACEAE } \\
\hline Xylopia arrmatica (Lam.) Mart. & - & - & - & - & 1,00 & 0,32 \\
\hline \multicolumn{7}{|l|}{ AQUIFOLIACEAE } \\
\hline Ilex affinis Gardner & $\begin{array}{c}26,0 \\
0\end{array}$ & $\begin{array}{c}3,3 \\
5\end{array}$ & 1,00 & 0,30 & $\begin{array}{c}12,0 \\
0\end{array}$ & 2,35 \\
\hline Ilex brevicusp is Reissek & - & - & 1,00 & 0,29 & 5,00 & 1,06 \\
\hline Ilex symplocifomis Rèssek & 9,00 & $\begin{array}{c}1,3 \\
3\end{array}$ & $\begin{array}{c}13,0 \\
0\end{array}$ & 2,92 & 5,00 & 1,71 \\
\hline Ilex theezans Mart. ex Reissek & 1,00 & $\begin{array}{c}0,1 \\
8\end{array}$ & 2,00 & 0,39 & 1,00 & 0,28 \\
\hline \multicolumn{7}{|l|}{ ARALIACEAE } \\
\hline Schefflera angustissina (Marchal) Frodin & 8,00 & $\begin{array}{c}1,1 \\
7\end{array}$ & 1,00 & 0,39 & 3,00 & 0,98 \\
\hline \multicolumn{7}{|l|}{ ASTERACEAE } \\
\hline Baccharis elliptica Gardner & $\begin{array}{c}53,0 \\
0\end{array}$ & $\begin{array}{c}5,1 \\
5\end{array}$ & 8,00 & 1,65 & - & - \\
\hline Baccharis retusa DC. & 4,00 & $\begin{array}{c}0,6 \\
1\end{array}$ & 1,00 & 0,30 & - & - \\
\hline Baccharis sp.1 & - & - & 3,00 & 0,69 & - & - \\
\hline Baccharis sp 2 & - & - & 1,00 & 0,33 & - & - \\
\hline Baccharis sp 3 & - & - & 2,00 & 0,42 & 1,00 & 0,29 \\
\hline Baccharis tridentata Baker & - & - & 2,00 & 0,83 & 9,00 & 2,21 \\
\hline Eremanthus erythropappus (DC.) MacLeish & $\begin{array}{c}14,0 \\
0\end{array}$ & $\begin{array}{c}3,6 \\
6\end{array}$ & 8,00 & 1,93 & 2,00 & 1,63 \\
\hline Lychonophora sp. & $\begin{array}{c}15,0 \\
0\end{array}$ & $\begin{array}{c}3,4 \\
5\end{array}$ & - & - & - & - \\
\hline Stenocephalum meg apotamicum (Spreng.) Sch.Bip. & 1,00 & $\begin{array}{c}0,1 \\
8\end{array}$ & - & - & - & - \\
\hline Trichogonia sp. & 1,00 & $\begin{array}{c}0,1 \\
8\end{array}$ & - & - & - & - \\
\hline $\begin{array}{l}\text { Vernonanthura polyan thes (Sprengel) Vega \& } \\
\text { Dematteis }\end{array}$ & 1,00 & $\begin{array}{l}0,1 \\
8\end{array}$ & 2,00 & 0,39 & - & - \\
\hline Vernonia sp. 1 & 2,00 & $\begin{array}{c}0,3 \\
6\end{array}$ & - & - & - & - \\
\hline Vernonia sp. 2 & - & - & - & - & 1,00 & 0,28 \\
\hline
\end{tabular}

BIGNONIACEAE

Handroanthus ochraceus (Cham.) Mattos

Jacaranda caroba (Vell) A.DC

BORAGINACEAE

Cordia trichotoma (Vell.) Arrab. ex Steud. 


\begin{tabular}{|c|c|c|c|c|c|c|c|c|c|c|c|c|c|}
\hline \multirow{3}{*}{ Família/Espécies } & \multicolumn{2}{|c|}{ Ambiente I } & \multirow{2}{*}{\multicolumn{2}{|c|}{ Ambiente II }} & \multicolumn{2}{|c|}{$\underset{\text { III }}{\text { Ambiente }}$} & \multirow{3}{*}{ Família/Espécies } & \multirow{2}{*}{\multicolumn{2}{|c|}{ Ambiente I }} & \multirow{2}{*}{\multicolumn{2}{|c|}{ Ambiente II }} & \multicolumn{2}{|c|}{$\underset{\text { III }}{\text { Ambiente }}$} \\
\hline & & & & & & & & & & & & & \\
\hline & NI & IVI & NI & IVI & NI & IVI & & NI & IVI & NI & IVI & NI & IVI \\
\hline Protium spruceamum (Benth.) Engl. & - & - & 1,00 & 2,33 & 1,00 & 0,38 & Byrsonima variabilis AJuss. & $\begin{array}{c}60,0 \\
0\end{array}$ & $\begin{array}{c}7,5 \\
6\end{array}$ & $\begin{array}{c}11,0 \\
0\end{array}$ & 2,89 & $\begin{array}{c}11,0 \\
0\end{array}$ & 3,85 \\
\hline CALOPHYLLACEAE & & & & & & & Byrsonima verbascifolia (L.) DC. & - & - & 1,00 & 0,32 & - & - \\
\hline Kielmeyera lathrophyton Saddi & 8,00 & $\begin{array}{c}1,4 \\
2\end{array}$ & $\begin{array}{c}12,0 \\
0\end{array}$ & 3,08 & 1,00 & 0,31 & Heteropterys sp. & - & - & - & - & 1,00 & 0,28 \\
\hline CELASTRACEAE & & & & & & & MALVACEAE & & & & & & \\
\hline Maytenus evonymoides Reissek & 7,00 & $\begin{array}{c}0,9 \\
8\end{array}$ & - & - & 5,00 & 1,63 & Eriotheca gracilipes (K.Schum.) A.Robyns & 2,00 & $\begin{array}{c}0,5 \\
9\end{array}$ & - & - & - & - \\
\hline CHR YSOBALANACEAE & & & & & & & MELASTOMATACEAE & & & & & & \\
\hline Hirtella glandulosa Spreng. & 9,00 & $\begin{array}{c}1,0 \\
9\end{array}$ & - & - & 1,00 & 0,29 & Lavoisiera imbricata (Thunb.) DC. & $\begin{array}{c}35,0 \\
0\end{array}$ & $\begin{array}{c}3,9 \\
5\end{array}$ & $\begin{array}{c}16,0 \\
0\end{array}$ & 3,04 & 9,00 & 4,57 \\
\hline CLUSIACEAE & & & & & & & Marcetia taxifolia (A.St.0Hil.) DC. & $\begin{array}{c}50,0 \\
0\end{array}$ & $\begin{array}{c}4,6 \\
6\end{array}$ & $\begin{array}{c}22,0 \\
0\end{array}$ & 3,45 & 7,00 & 1,39 \\
\hline Clusia cruvva Cambess. & 4,00 & $\begin{array}{c}0,8 \\
0\end{array}$ & 5,00 & 1,27 & 1,00 & 1,26 & Miconia albicans (Sw.)Triana & - & - & 1,00 & 0,45 & 3,00 & 0,48 \\
\hline EBENACEAE & & & & & & & Miconia cinerascens Miq. & 1,00 & $\begin{array}{c}0,3 \\
2\end{array}$ & 1,00 & 0,47 & 6,00 & 1,35 \\
\hline Diospyros hispida A.DC. & - & - & - & - & 1,00 & 0,28 & Miconia corallina Spring & 1,00 & $\begin{array}{c}0,1 \\
8\end{array}$ & 2,00 & 0,59 & $\begin{array}{c}13,0 \\
0\end{array}$ & 2,63 \\
\hline Diospyros inconstans Jacq. & 1,00 & $\begin{array}{c}0,1 \\
8\end{array}$ & 2,00 & 0,89 & $\begin{array}{c}12,0 \\
0\end{array}$ & 2,14 & Miconia latecrenata (DC) Naudin & - & - & 6,00 & 1,73 & 2,00 & 0,61 \\
\hline ERYTROXYLACEAE & & & & & & & Miconia pepericarpa DC. & - & - & - & - & 3,00 & 2,56 \\
\hline Erythroxylum cuneifolium (Mart.) O.ESchulz & 4,00 & $\begin{array}{c}0,7 \\
7\end{array}$ & 3,00 & 0,91 & 1,00 & 0,28 & Miconia sp.1 & $\begin{array}{c}10,0 \\
0\end{array}$ & $\begin{array}{c}1,4 \\
4\end{array}$ & 1,00 & 0,31 & $\begin{array}{c}10,0 \\
0\end{array}$ & 2,68 \\
\hline Erythroxylum pelleteriamum A.St.0Hil. & 1,00 & $\begin{array}{c}0,1 \\
9\end{array}$ & - & - & 2,00 & 0,56 & Miconia sp 2 & - & - & 1,00 & 0,29 & - & - \\
\hline EUPHORBIACEAE & & & & & & & Miconia sp 3 & 6,00 & $\begin{array}{c}1,0 \\
8\end{array}$ & 2,00 & 0,60 & 3,00 & 0,69 \\
\hline Croton sp. & 5,00 & $\begin{array}{c}0,7 \\
7\end{array}$ & 1,00 & 0,30 & - & - & Miconia tristis Spring & 2,00 & $\begin{array}{c}0,8 \\
1\end{array}$ & - & - & 1,00 & 0,57 \\
\hline Sapium glandulosum (L.) Morong & 1,00 & $\begin{array}{c}0,1 \\
8\end{array}$ & - & - & - & - & Microlicia isophylla DC. & $\begin{array}{c}22,0 \\
0\end{array}$ & $\begin{array}{c}2,6 \\
5\end{array}$ & $\begin{array}{c}19,0 \\
0\end{array}$ & 2,91 & 4,00 & 1,38 \\
\hline Sapium sp. & $\begin{array}{c}44,0 \\
0\end{array}$ & $\begin{array}{c}6,8 \\
8\end{array}$ & $\begin{array}{c}18,0 \\
0\end{array}$ & 3,64 & 7,00 & 1,65 & Pleroma fothergillii (Schrank et Mat. ex DC.) Triana & $\begin{array}{c}13,0 \\
0\end{array}$ & $\begin{array}{c}1,8 \\
9\end{array}$ & $\begin{array}{c}26,0 \\
0\end{array}$ & 4,35 & $\begin{array}{c}29,0 \\
0\end{array}$ & 5,85 \\
\hline Sebastiania sp. & $\begin{array}{c}19,0 \\
0\end{array}$ & $\begin{array}{c}2,6 \\
0\end{array}$ & 1,00 & 0,30 & 1,00 & 0,28 & Tibouchina estrellensis (Raddi) Cogn. & - & - & 1,00 & 0,30 & 7,00 & 1,15 \\
\hline FABACEAE & & & & & & & Tibouchina sellowiana $\operatorname{Cog} \mathrm{n}$. & 1,00 & $\begin{array}{c}0,1 \\
7\end{array}$ & 2,00 & 0,39 & 1,00 & 5,87 \\
\hline Bauhinia sp. & 1,00 & $\begin{array}{c}0,1 \\
7\end{array}$ & - & - & - & - & Trembleya lanifora (D.Don) Cogn. & $\begin{array}{c}58,0 \\
0\end{array}$ & $\begin{array}{c}8,6 \\
5\end{array}$ & $\begin{array}{c}45,0 \\
0\end{array}$ & $\begin{array}{c}14,6 \\
4\end{array}$ & $\begin{array}{c}14,0 \\
0\end{array}$ & 6,91 \\
\hline Calliandra sp. & 2,00 & $\begin{array}{c}0,2 \\
3\end{array}$ & - & - & - & - & MELIACEAE & & & & & & \\
\hline Copaifera langsdorffii Desf. & 7,00 & $\begin{array}{c}0,8 \\
7\end{array}$ & $\begin{array}{c}15,0 \\
0\end{array}$ & 2,20 & - & - & Cabralea canjerana (Vell.) Mart. & 1,00 & $\begin{array}{c}0,1 \\
8\end{array}$ & 1,00 & 0,30 & 3,00 & 1,01 \\
\hline Diplotropisfernu ginea Benth. & - & - & - & - & 2,00 & 0,39 & Trichilia elegans A.Juss. & - & - & - & - & 2,00 & 0,55 \\
\hline Swartzia apetala Raddi & - & - & - & - & 2,00 & 0,55 & MYRTACEAE & & & & & & \\
\hline GENTIANACEAE & & & & & & & Blepharocalyx salicifolius (Kunth) O.Berg & 2,00 & $\begin{array}{c}0,2 \\
2\end{array}$ & - & - & 1,00 & 0,28 \\
\hline Calolisianthus sp. & 1,00 & $\begin{array}{c}0,1 \\
8\end{array}$ & - & - & - & - & Calyptranthes sp. & 2,00 & $\begin{array}{c}0,4 \\
7\end{array}$ & - & - & - & - \\
\hline HYPERICACEAE & & & & & & & Campomanesia guazumifolia (Cambess.) O.Berg & - & - & - & - & 1,00 & 0,28 \\
\hline Vismia guianensis (Aubl.) Pers. & - & - & 3,00 & 0,69 & - & - & Campomanesia xanthocarpa (Mart.) O.Berg & 2,00 & $\begin{array}{c}0,3 \\
6\end{array}$ & - & - & - & - \\
\hline LAMIACEAE & & & & & & & Eugenia aurata O.Berg & 4,00 & $\begin{array}{c}0,8 \\
0\end{array}$ & 5,00 & 1,09 & - & - \\
\hline Aegiphila verticillata Vell. & 2,00 & $\begin{array}{c}0,4 \\
5\end{array}$ & - & - & - & - & Eugenia punicifolia (Kunth) DC. & 3,00 & $\stackrel{0,3}{1}$ & 1,00 & 0,29 & 1,00 & 0,28 \\
\hline LAURACEAE & & & & & & & Eugenia ramboi DLegrand & 8,00 & $\begin{array}{c}3,9 \\
3\end{array}$ & - & - & - & - \\
\hline Ocotea bicolor Vattimo0Gil & 9,00 & $\begin{array}{c}1,3 \\
4\end{array}$ & 3,00 & 0,47 & $\begin{array}{c}15,0 \\
0\end{array}$ & 3,05 & Eugenia sonderiana $\mathrm{O} . \mathrm{Berg}$ & 3,00 & $\stackrel{0,5}{2}$ & 4,00 & 0,79 & - & - \\
\hline Ocotea elegans Mez & - & - & 2,00 & 0,78 & 1,00 & 0,28 & Eugenia sp. & 5,00 & $\stackrel{0,8}{5}$ & - & - & - & - \\
\hline Ocotea pulchella (Nees \& Mart.) Mez & 9,00 & $\begin{array}{c}1,3 \\
3\end{array}$ & 5,00 & 1,03 & 3,00 & 0,83 & Marlierea laevigata (DC.) Kiaersk. & - & - & - & - & 3,00 & 1,93 \\
\hline LOGANIACEAE & & & & & & & Marlierea racemosa (Vell.) Kaersk. & 3,00 & $\begin{array}{c}0,3 \\
6\end{array}$ & 4,00 & 1,36 & 3,00 & 0,72 \\
\hline Strychnos sp. & - & - & - & - & 1,00 & 0,28 & Myrcia guianensis (Aubl.) DC. & 1,00 & $\begin{array}{c}0,1 \\
9\end{array}$ & $\begin{array}{c}12,0 \\
0\end{array}$ & 1,40 & - & - \\
\hline MALPIGHIACEAE & & & & & & & Myrcia hartwegiana (O.Berg) Kiaesk. & 5,00 & $\begin{array}{c}1,0 \\
2\end{array}$ & - & - & - & - \\
\hline Banisteriopsis sp. & - & - & - & - & 1,00 & 0,28 & Myrcia hebepetala DC. & - & - & 2,00 & 0,59 & - & - \\
\hline Banisteriopsis stellaris (Griseb.) B.Gates & 2,00 & $\begin{array}{c}0,4 \\
8\end{array}$ & - & - & 2,00 & 0,59 & Myrcia oblongata DC. & 4,00 & $\begin{array}{c}0,5 \\
0\end{array}$ & - & - & - & $\cdot$ \\
\hline Byrsonima intermedia $\mathrm{A}$. Juss. & 3,00 & $\begin{array}{c}0,4 \\
1\end{array}$ & 6,00 & 1,16 & 1,00 & 0,28 & Myrcia sp. & 4,00 & $\begin{array}{c}0,4 \\
6\end{array}$ & - & - & 1,00 & 0,30 \\
\hline Byrsonima pachyphylla AJuss. & 1,00 & $\begin{array}{c}0,1 \\
8\end{array}$ & 1,00 & 0,30 & 2,00 & 0,59 & Myrcia splendens (Sw.) DC. & 3,00 & $\begin{array}{c}0,5 \\
3\end{array}$ & $\begin{array}{c}11,0 \\
0\end{array}$ & 1,60 & 4,00 & 1,15 \\
\hline
\end{tabular}


Oliveira et al.

\begin{tabular}{|c|c|c|c|c|c|c|}
\hline \multirow{2}{*}{ Família/Espécies } & \multicolumn{2}{|c|}{ Ambiente I } & \multicolumn{2}{|c|}{ Ambiente II } & \multicolumn{2}{|c|}{$\begin{array}{c}\text { Ambiente } \\
\text { III }\end{array}$} \\
\hline & NI & IVI & NI & IVI & NI & IVI \\
\hline Myrcia tomentosa (Aub1.) DC. & 6,00 & $\begin{array}{c}0,7 \\
3\end{array}$ & - & - & - & - \\
\hline Myrcia venulosa DC. & 3,00 & $\begin{array}{c}0,5 \\
0\end{array}$ & - & - & - & - \\
\hline Myrciaria sp. & - & - & - & - & 1,00 & 0,28 \\
\hline Psidium rufum $\mathrm{DC}$. & - & - & - & - & 1,00 & 0,30 \\
\hline Psidium sp. & 1,00 & $\begin{array}{c}0,1 \\
9\end{array}$ & - & - & - & - \\
\hline Siphonaugena densiffora O.Berg & - & - & - & - & 1,00 & 0,30 \\
\hline Siphonaugena sp. & - & - & 1,00 & 0,29 & - & - \\
\hline \multicolumn{7}{|l|}{ NYCTAGINACEAE } \\
\hline Guapira graciliflora (Mart. ex Schmidt) Lundell & - & - & 1,00 & 0,30 & 5,00 & 1,13 \\
\hline Guapira opposita (Vell.) Reitz & - & - & 3,00 & $\underset{5}{10,9}$ & 1,00 & 0,43 \\
\hline Guapira venosa (Choisy) Lundell & 6,00 & $\stackrel{0,8}{3}$ & - & - & - & - \\
\hline \multicolumn{7}{|l|}{ OCHNACEAE } \\
\hline Luxemburgia schwackeana Taub. & 3,00 & $\stackrel{0,3}{9}$ & - & - & - & - \\
\hline \multicolumn{7}{|l|}{ OLEACEAE } \\
\hline Chionanthus crassifolius (Mart.) PS. Green & - & - & 1,00 & 0,31 & 1,00 & 0,31 \\
\hline \multicolumn{7}{|l|}{ PHYLLANTHACEAE } \\
\hline Margaritaria nobilis L.f. & - & - & - & - & 1,00 & 0,28 \\
\hline \multicolumn{7}{|l|}{ POLYGONACEAE } \\
\hline Coccoloba mollis Casar. & - & - & - & - & 3,00 & 0,67 \\
\hline \multicolumn{7}{|l|}{ PRIMULACEAE } \\
\hline Myrsine coriacea (Sw.) R.Br. ex Roem. \& Schult. & 1,00 & $\frac{0,2}{6}$ & - & - & - & - \\
\hline Myrsine guianensis (Aubl.) Kuntze & 3,00 & $\begin{array}{c}0,4 \\
0\end{array}$ & $\begin{array}{c}12,0 \\
0\end{array}$ & 2,51 & 3,00 & 0,79 \\
\hline Myrsine lineata (Mez) Imkhan. & - & - & 1,00 & 0,30 & 2,00 & 0,39 \\
\hline \multicolumn{7}{|l|}{ PROTEACEAE } \\
\hline Roupala montana Aubl. & - & - & 3,00 & 1,68 & 5,00 & 4,01 \\
\hline \multicolumn{7}{|l|}{ RUBIACEAE } \\
\hline Amaioua guianensis Aubl. & 1,00 & $\stackrel{0,1}{8}$ & 3,00 & 0,49 & $\underset{0}{15,0}$ & 2,9 \\
\hline Cordiera concolor (Cham.) Kuntze & - & - & - & - & 4,00 & 0,95 \\
\hline Hamelia patens Jacq. & 5,00 & $\stackrel{0,5}{3}$ & 7,00 & 1,51 & 6,00 & 1,34 \\
\hline Palicourea rigida Kunth & $\begin{array}{c}27,0 \\
0\end{array}$ & $\begin{array}{c}4,1 \\
2\end{array}$ & 9,00 & 2,20 & 1,00 & 0,28 \\
\hline Posoqueria sp. & 3,00 & $\begin{array}{c}0,6 \\
4\end{array}$ & 7,00 & 1,14 & 2,00 & 0,65 \\
\hline Psychotria vellosiana Benth. & - & - & 3,00 & 0,70 & $\begin{array}{c}17,0 \\
0\end{array}$ & 2,51 \\
\hline Remijia ferruginea (A.St.-Hil.)DC. & 9,00 & $\begin{array}{c}1,0 \\
0\end{array}$ & - & - & - & - \\
\hline \multicolumn{7}{|l|}{ SALICACEAE } \\
\hline Casearia sylestris Sw. & 1,00 & $\stackrel{0,1}{8}$ & 3,00 & 0,71 & 1,00 & 0,28 \\
\hline \multicolumn{7}{|l|}{ SAPINDACEAE } \\
\hline Averrhoidium gardnerianum Baill. & 6,00 & $\begin{array}{c}0,7 \\
8\end{array}$ & - & - & - & - \\
\hline Cupania vemalis Cambess. & 2,00 & $\begin{array}{l}1,1 \\
7\end{array}$ & - & - & 1,00 & 0,28 \\
\hline \multicolumn{7}{|l|}{ SAPOTACEAE } \\
\hline Pouteria gandneri (Mart. \& Miq.) Bæhni & 3,00 & $\stackrel{0,5}{4}$ & - & - & - & - \\
\hline \multicolumn{7}{|l|}{ STYRACACEAE } \\
\hline Styrax camporum Pohl & 6,00 & 1,4 & - & - & 1,00 & 0,53 \\
\hline
\end{tabular}

\begin{tabular}{|c|c|c|c|c|c|c|}
\hline \multirow{2}{*}{ Família/Espécies } & \multicolumn{2}{|c|}{ Ambiente I } & \multicolumn{2}{|c|}{ Ambiente II } & \multicolumn{2}{|c|}{$\begin{array}{c}\text { Ambiente } \\
\text { III }\end{array}$} \\
\hline & NI & IVI & NI & IVI & NI & IVI \\
\hline \multicolumn{7}{|l|}{ SYMPLOCACEAE } \\
\hline Symplocos celastrinea Mart. & - & - & - & - & 3,00 & 0,48 \\
\hline Symplocos oblong ifolia Casar. & - & - & - & - & 1,00 & 0,29 \\
\hline Symplocos sp. & - & - & - & - & 6,00 & 0,97 \\
\hline \multicolumn{7}{|l|}{ VERBENACEAE } \\
\hline Lantana sp. & - & - & 4,00 & 1,17 & 1,00 & 0,32 \\
\hline \multicolumn{7}{|l|}{ VOCHYSIACEAE } \\
\hline Vochysia tucanorum Mart. & - & - & 1,00 & 0,45 & 2,00 & 1,42 \\
\hline \multicolumn{7}{|l|}{ WINTERACEAE } \\
\hline Drimys brasiliensis Miers & - & - & - & - & 1,00 & 0,28 \\
\hline \multicolumn{7}{|l|}{ NÃO IDENTIFICADA } \\
\hline NÃO IDENTIFICADA & - & - & 1,00 & 0,65 & - & - \\
\hline
\end{tabular}

As espécies com maior IVI foram Trembleya laniflora (D. Don) Cogn. (Melastomataceae), seguida de Byrsonima variabilis A. Juss. (Malpighiaceae), Sapium sp. (Euphorbiaceae), Pleroma fothergillii (Schrank et Mat. ex DC.) Triana (Melastomataceae), e Lavoisiera imbricata (Thunb.) DC. (Melastomataceae), estiveram presentes em ambos os ambientes.

No ambiente III foi registrado o maior número de espécies (Figura 1). Entre as espécies registradas 26\% foram comuns aos três ambientes (Figura 1). O ambiente I teve o menor número de espécies compartilhadas com os demais, sugerindo que a divisão entre os ambientes diferenciou corretamente os ambientes. Pelo índice de similaridade de Jaccard, apenas os ambientes II e III apresentaram elevada similaridade. De maneira geral a comunidade apresentou valor de Shannon para a diversidade de 2,48. O ambiente III deteve a mais elevada diversidade de espécies, seguido do ambiente I, e do ambiente II pelo teste t de Hutcheson (Figura $1)$.

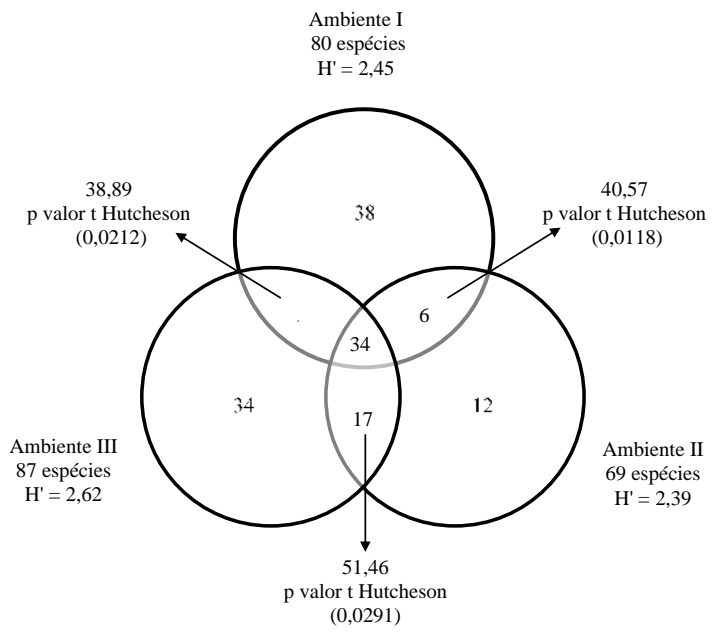

Figura 1. Diagrama de Venn extraído da composição florística de diferentes ambientes de uma área degradada por voçorocamento no Parque Estadual do Biribiri, Diamantina, $\mathrm{MG}$, demonstrando a diversidade de Shannon ( $\left.\mathrm{H}^{\prime}\right)$, o número de espécies exclusivas e comuns entre os estratos e Índice de 
similaridade de Jaccard e entre parênteses os valores de p para o teste $\mathrm{t}$ de Hutcheson.

O dendrograma gerado pela análise de agrupamentos (Figura 2) evidencia uma separação das parcelas em dois grandes grupos, o primeiro, formado pelas parcelas do ambiente I e parte das parcelas do ambiente II. Já o segundo, foi formado pelas parcelas do ambiente III e parte do ambiente II. No ambiente II, são encontradas tanto espécies colonizadoras inicias encontradas no ambiente I, quanto espécies do ambiente III (Tabela 2).

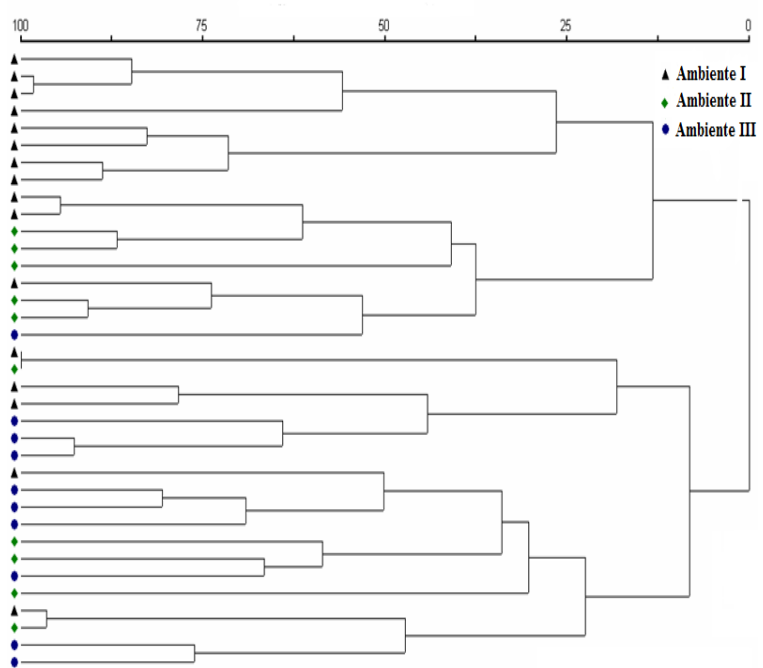

Figura 2. Dendrograma de similaridade produzido pela análise de agrupamento da composição de espécies de três ambientes de uma voçoroca no Parque Estadual do Biribiri, Diamantina, MG.

Das 131 espécies analisadas pelo método das espécies indicadoras (Tabela 3), apenas dez (7\%) apresentaram preferência significativa por um dos habitats de solo, sendo 2 $(1,5 \%)$ pelo ambiente I, $1(0,7 \%)$ pelo ambiente II e $7(5,4 \%)$ pelo ambiente III.

Tabela 3. Análise de espécie indicadoras (ISA) realizada com base nos habitats de solo e valor de cobertura das 131 espécies. $\mathrm{VIO}=$ valor indicador observado, $\mathrm{VIE}=$ valor indicador esperado (média, desvio padrão e significância) e abundância relativa de indivíduos em cada habitat de solo: I = Ambiente I; II = Ambiente II; III = Ambiente III

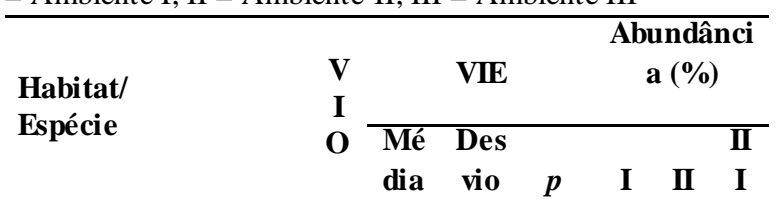

\section{Ambiente}

I

\begin{tabular}{|c|c|c|c|c|c|c|c|}
\hline $\begin{array}{l}\text { Symplocos lanceolata } \\
\text { (Mart.) A.DC. }\end{array}$ & $\begin{array}{l}31 \\
, 2\end{array}$ & $\begin{array}{c}14 \\
5\end{array}$ & 7,1 & $\begin{array}{r}0,0 \\
382\end{array}$ & $\begin{array}{c}10 \\
0\end{array}$ & 0 & 0 \\
\hline $\begin{array}{l}\text { Miconia latecrenata } \\
\text { (DC.) Naudin }\end{array}$ & $\begin{array}{r}37 \\
, 4\end{array}$ & $\begin{array}{c}19 \\
0\end{array}$ & 7,5 & $\begin{array}{r}0,0 \\
300\end{array}$ & 86 & 7 & 7 \\
\hline
\end{tabular}

Ambiente

\section{II}

$\begin{array}{lrccccccc}\text { Vochysia tucanorum } & 37 & 15, & 6,6 & 0,0 & 0 & 7 & 25 \\ \text { Mart. } & , 5 & 9 & & 160 & & 5 & \end{array}$

\section{Ambiente}

III

\begin{tabular}{|c|c|c|c|c|c|c|c|}
\hline $\begin{array}{l}\text { Amaioua guianensis } \\
\text { Aubl. }\end{array}$ & $\begin{array}{r}40 \\
, 3\end{array}$ & $\begin{array}{c}17 \\
0\end{array}$ & 7,6 & $\begin{array}{l}0,0 \\
168\end{array}$ & 3 & $\begin{array}{l}1 \\
6\end{array}$ & 81 \\
\hline $\begin{array}{l}\text { Guapira venosa } \\
\text { (Choisy) Lundell }\end{array}$ & $\begin{array}{r}30 \\
, 0\end{array}$ & $\begin{array}{c}10 \\
8\end{array}$ & 6,0 & $\begin{array}{r}0,0 \\
350\end{array}$ & 0 & 0 & $\begin{array}{c}10 \\
0\end{array}$ \\
\hline $\begin{array}{l}\text { Maytenus } \\
\text { evonymoides Reissek }\end{array}$ & $\begin{array}{r}41 \\
, 0\end{array}$ & $\begin{array}{c}18 \\
0\end{array}$ & 7,5 & $\begin{array}{r}0,0 \\
208\end{array}$ & 4 & $\begin{array}{l}1 \\
4\end{array}$ & 82 \\
\hline $\begin{array}{l}\text { Tibouchina } \\
\text { estrellensis Cogn }\end{array}$ & $\begin{array}{r}30 \\
, 0\end{array}$ & $\begin{array}{c}10 \\
4\end{array}$ & 6,2 & $\begin{array}{r}0,0 \\
342\end{array}$ & 0 & 0 & $\begin{array}{c}10 \\
0\end{array}$ \\
\hline $\begin{array}{l}\text { Averrhoidium } \\
\text { garnerianum Baill }\end{array}$ & $\begin{array}{r}30 \\
, 0\end{array}$ & $\begin{array}{c}10 \\
5\end{array}$ & 6,2 & $\begin{array}{r}0,0 \\
358\end{array}$ & 0 & 0 & $\begin{array}{c}10 \\
0\end{array}$ \\
\hline $\begin{array}{l}\text { Vismia guianensis } \\
\text { (Aubl.) Pers. }\end{array}$ & $\begin{array}{l}41 \\
, 6\end{array}$ & $\begin{array}{c}17 \\
5\end{array}$ & 7,3 & $\begin{array}{l}0,0 \\
066\end{array}$ & 4 & $\begin{array}{l}1 \\
3\end{array}$ & 83 \\
\hline Hamelia patens Jacq & $\begin{array}{r}50 \\
, 8\end{array}$ & $\begin{array}{c}25, \\
2\end{array}$ & 7,5 & $\begin{array}{r}0,0 \\
074\end{array}$ & 24 & $\begin{array}{l}1 \\
3\end{array}$ & 63 \\
\hline
\end{tabular}

\section{Discussão}

As famílias com maior riqueza de espécies Myrtaceae, Melastomataceae, Asteraceae, Rubiaceae e Malpighiaceae se assemelham às encontradas por Amaral et al. (2013) em área degradada pela extração de ouro, que se encontra em processo de recuperação por meio da regeneração natural, no Parque Estadual do Biribiri.

A família Myrtaceae apresenta grande importância nas florestas brasileiras sendo registrada com maior riqueza em diversas fisionomias brasileiras como: Floresta Estacional Semidecidual Montana (Gonzaga et al. 2008) Floresta Atlântica Montana (Padgurschi et al. 2011), Mata de Restinga Arenosa (Santos et al. 2012), Floresta Ombrófila Densa de planície em processo de recuperação (Nascimento et al. 2012) e Cerrado Rupestre (Amaral et al. 2013). Mostrando assim a importância das mesmas na colonização da área de estudo.

O maior número de espécies presente no ambiente III pode estar relacionado com as condições edáficas do local. Este se encontra localizado na parte superior da voçoroca, com solos mais profundos, presença de maior teor de matéria orgânica e argila. Segundo Budowski (1966) há menor número de espécies nas primeiras fases de sucessão o que sugere que o ambiente III encontra-se em fase mais avançada de sucessão.

Segundo a hipótese do distúrbio intermediário o número de espécies está relacionado com a intensidade dos transtornos causados (Connel, 1978). Em baixa intensidade de modificações, as espécies competidoras tendem a dominar o local. Sob alta intensidade apenas as espécies tolerantes são capazes de sobreviverem à perturbação. Assim, acredita-se que o ambiente III sofreu um distúrbio intermediário, o que levou ao maior acréscimo no número de espécies locais, pois, tanto as espécies tolerantes quanto as dominantes foram capazes de coexistirem (Connel, 1978; Huston, 1979; Shea et al. 2004).

De maneira geral a comunidade apresentou baixa diversidade $\left(\mathrm{H}^{\prime}=2,483\right)$ quando comparada a área de cerrado rupestre próxima ( $\mathrm{H}^{\prime}$ de 2,742 a 3,921), a qual se encontra em regeneração natural após a degradação pela extração de ouro (Amaral et al. 2013). Porém, a mesma foi considerada elevada quando comparada a diferentes ambientes de uma cascalheira 
em recuperação ( $\mathrm{H}^{\prime}$ de 1,680 a 2,074), também no Parque Estadual do Biribiri (Pereira et al. 2015).

$\mathrm{O}$ reduzido número de espécies indicadoras pode estar relacionado com a elevada proximidade entre os ambientes que pode ter favorecido o compartilhamento de elementos da flora (Amaral et al. 2013). Distância entre os fragmentos, estágio sucessional, grau de isolamento e o bioma predominante ao qual o remanescente está inserido, podem comprometer a classificação das espécies como indicadoras (Almeida e Machado, 2007).

\section{Conclusão}

As espécies Symplocos laceotala e Miconia latecrenata foram indicadoras do ambiente em fase inicial de sucessão devendo ser usadas no processo inicial de recuperação de áreas degradadas. Já a espécie Vochysia tucanorum foi indicadora da fase intermediária de sucessão. Por outro lado, as espécies Amaioua guianensis, Guapira venosa, Maytenus evonymoides, Tibouchina estrellensis, Averrhoidium garnerianum, Vismia guianensis e Hamelia patens devem ser usadas na fase final da recuperação de áreas degradadas, por terem sido classificadas como indicadoras do ambiente em estágio avançado de sucessão.

\section{Referências}

Abdo M, Vieira SR, Martins ALM, Silveira LCP (2008) Estabilização de uma voçoroca no pólo Apta centro Norte Pindorama, SP. Revista Tecnologia \& Inovação Agropecuária, 1(1): 135-141.

Almeida HS, Machado ELM (2007) Espécies Indicadoras do Componente Arbóreo em Comunidades de Floresta Estacional Decídua. Revista Brasileira de Biociências, 5(S1): 654-656.

Amaral WG, Pereira IM, Amaral CS, Machado ELM, Rabelo LDO (2013) Dinâmica da flora arbustivo-arbórea colonizadora em uma área degradada pela extração de ouro em Diamantina, MG. Ciência Florestal, 23(4): 713-725. doi:10.5902/1980509812355

APG IV (2016) Angiosperm Phy logeny Group. An update of the Angiosperm Phylogeny Group classification for the orders and families of flowering plants. Botanical Journal of the Linnean Society, 181(1): 1-20. doi:10.1111/boj.12385

Bonini I, Daltro RF, Ribeiro RQA (2013) Influência da supressão vegetal na dinâmica de processos erosivos: um estudo comparativo em Campo Verde, Mato Grosso. Enciclopédia Biosfera, Centro Científico Conhecer, 9(16): 2122-2135.

Brower JE, Zar JH (1984) Field and laboratory methods for general ecology. Dubuque, W.M.C. Brow, 84 p.

Budowski G (1966) Distribution of tropical American rain forest species in the light of successional processes. Turrialba, 15: 40-42.

Carneiro MAC, Siqueira JO, Moreira FMS, Soares ALL (2009) Atributos físicos, químicos e biológicos de solo de cerrado sob diferentes sistemas de uso e manejo. Revista Brasileira de Ciência do solo, 33(1): 174-157.

Connell, JH (1978) Diversity in tropical rain forests and coral reefs. $\quad$ Science, 199(4335): 1302-1310. doi:10.1126/science.199.4335.1302.
Dufrêne M, Legendre, P (1997) Species assemblages and indicator species: the need for a flexible asymmetrical approach. Ecological monographs, 67(3): 345-366. doi:10.1890/00129615(1997)067[0345:SAAIST]2.0.CO;2

Echternacht L, Trovó M, Oliveira CT, Pirani JR (2011) Areas of endemism in the Espinhaço range in Minas Gerais, Brazil. Flora-Morphology, Distribution, Functional Ecology of Plants, 206(9), 782-791.

Ferreira J, Pardini R, Metzger JP, Fonseca CR, Pompeu PS, Sparovek G, Louzada J (2012) Towards environmentally sustainable agriculture in Brazil: challenges and opportunities for applied ecological research. Journal of Applied Ecology 49(3): 535-541. doi:10.1111/j.13652664.2012.02145.x.Ferreira VM, Silva MLN, Curi N, Oliveira AH, Silva MA, Avanzi JC (2011) Influência antrópica e atributos de solo: inter-relações em ambientes de voçorocas na mesorregião Campos das Vertentes, MG. Geografia, 36(1): 209-219.

Finol Urdaneta, H (1971) Nuevos parámetros a considerarse en el análisis estructural de las selvas vírgenes tropicales. Revista Forestal Venezolana, 14 (21): p. 29-42.

Gomide PHO, Silva MLN, Soares CRFS (2011) Atributos físicos, químicos e biológicos do solo em ambientes de voçorocas no município de Lavras-MG. Revista Brasileira de Ciência do solo, 35(2): 567-577.

Gonzaga APD, Oliveira-Filho AT, Machado ELM, Hargreaves P, Machado JNM (2008) Diagnóstico florístico-estrutural do componente arbóreo da floresta da Serra de São José, Tiradentes, MG, Brasil. Acta botanica brasilica, 22(2): 505-520.

Goulart MF, Azevedo, AA (2015) Trilha da Água Limpa: roteiro educativo no Parque Estadual do Biribiri.

Gutiérrez AG, Schanabel S, Contador FL (2009) Gully erosion, land use and topographical thresholds during the last 60 years in a small rangeland catchment in SW Spain. Land Degradation \& Development, 20(5): 535-550. doi:10.1002/ldr.931

Huston MA (1979) A general hy pothesis of species diversity. The American Naturalist, 113(1): 81-101. doi:10.1086/283366

IEF (2005) Instituto Estadual De Florestas. Plano de Manejo do Parque Estadual do Biribiri, 2005.

Lanza DA , Pott A, Silva J (2014) Vegetação e uso da Terra na Unidade de Planejamento e Gerenciamento Rio Verde, Mato Grosso do Sul. Revista GeoPantanal, 9(16): 251-262.

Loschi RA, Pereira JAA, Machado ELM, Carlos L, Sá JJG, Marques M (2011) Interações espécie-ambiente na colonização de uma voçoroca em Itumirim, Minas Gerais. Cerne, 17(2): 161-180.

Pereira IM, Gonzaga APD, Machado ELM, Oliveira MLR, Marques, IC (2015). Estrutura da vegetação colonizadora em ambiente degradado por extração de cascalho em Diamantina, MG. Pesquisa Florestal Brasileira, 35(82), 77 88. 
McCune B, Mefford MJ (1999) PC-ORD. Análise multivariada de dados ecológicos. Versão 3.12. Oregon, EUA.

Messias MCTB, Leite MGP, Meira-Neto JAA, Kozovits AR (2012) Fitossociologia de campos rupestres quartzíticos e ferruginosos no Quadrilátero Ferrífero, Minas Gerais. Acta Botanica Brasilica, 26(1): 230-242.

Nascimento LM, Sampaio EVSB, Rodal MJN, Silva SI, Silva ACBL (2012) Natural forest regeneration in abandoned sugarcane fields in northeastern Brazil: floristic changes. Biota Neotropica, 12(4): 84-97. doi:10.1590/S167606032012000400009

Padgurschi MCG, Pereira LP, Tamashiro JY, Joly CA (2011) Floristic composition and similaritie between areas of Montane Atlantic rainforest, São Paulo, Brazil. Biota Neotropica, 11(2): 139-152. doi:10.1590/S167606032011000200014

Pereira IM, Gonzaga APD, Machado ELM, Oliveira MLR, Marques IC (2015) Estrutura da vegetação colonizadora em ambiente degradado por extração de cascalho em Diamantina, MG. Pesquisa Florestal Brasileira, 35(82): 77 88. doi:10.4336/2015.pfb.35.82.769

Sano EE, Rodrigues AA, Martins ES, Bettiol GM, Bustamante MM, Bezerra AS, Bolfe EL(2019) Cerrado ecoregions: A spatial framework to assess and prioritize Brazilian savanna environmental diversity for conservation. Journal of environmental management, 232: 818-828. doi: 10.1016/j.jenvman.2018.11.108

Sano EE, Rosa R, Brito JLS, Ferreira LG (2010). Land cover mapping of the tropical savanna region in Brazil. Environmental Monitoring and Assessment 166(1-4): 113124. doi:10.1007/s10661-009-0988-4

Sá Júnior A, Carvalho LG, Silva FF, Alves MC (2012) Application of the Köppen classification for climatic zoning in the state of Minas Gerais, Brazil. Theoretical and Applied Climatology, 108(1-2): 1-7. doi:10.1007/s00704011-0507-8

Santos R, Silva RC, Pacheco D, Martins R, Citadini-Zanette V (2012) Florística e estrutura do componente arbustivoarbóreo de mata de restinga arenosa no Parque Estadual de Itapeva, Rio Grande do Sul. Revista Árvore, 36(6): 10471059

Shea K, Roxvurgh, H, Rauschert, E (2004). Moving from pattern to process: coexistence mechanisms under intermediate disturbance regimes. Ecology letters, 7(6): 491-508. doi:10.1111/j.1461-0248.2004.00600.x

Oliveros JC (2007) Venny. An interactive tool for comparing lists with Venn Diagrams. http://bioinfogp. cnb. csic. es/tools/venny/index. html.

Zar JH (1996) Biostatistical analy sis. Biostatistical Analy sis. 4th Edition, Practice Hall, New Jersey. 